\title{
All-optical reconstruction of atomic ground-state population
}

\author{
P. London, ${ }^{1}$ O. Firstenberg, ${ }^{1}$ M. Shuker ${ }^{1}$ and A. Ron ${ }^{1}$ \\ ${ }^{1}$ Department of Physics, Technion-Israel Institute of Technology, Haifa 32000, Israel
}

\begin{abstract}
The population distribution within the ground-state of an atomic ensemble is of large significance in a variety of quantum optics processes. We present a method to reconstruct the detailed population distribution from a set of absorption measurements with various frequencies and polarizations, by utilizing the differences between the dipole matrix elements of the probed transitions. The technique is experimentally implemented on a thermal rubidium vapor, demonstrating a population-based analysis in two optical pumping examples. The results are used to verify and calibrate an elaborated numerical model, and the limitations of the reconstruction scheme which result from the symmetry properties of the dipole matrix elements are discussed.
\end{abstract}

\section{INTRODUCTION}

In the past decades, alkali-metal atoms have become a central ingredient in quantum optics applications, mostly due to the simplicity of their electronic internal structure. In many atom-photon phenomena and particularly in multi-photon processes, a key element is the distribution of populations among the various states within the alkali ground-level manifold. As an example, consider the implementation of a frequency reference based on coherent population trapping (CPT), in which two resonant fields are used to create and interrogate the coherence between two specific ground-level states [1, 2]. Dilution of the CPT states due to optical pumping and the redistribution of population towards highly polarized states pose a major limit on the CPT contrast [3]. In addition, schemes for narrow CPT resonances have been proposed in which spin-exchange decay is suppressed by the use of a proper population distribution [4]. For such realizations of CPT and similar physical arrangements, the detailed electronic population distribution is of great importance.

Population reconstruction is a measurement of the projection of an unknown density matrix along a certain quantization axis (diagonal elements). If similar measurements are carried out from sufficient directions, a tomographic reconstruction of the full density matrix (offdiagonal elements) is possible [5]. Various reconstruction schemes have been demonstrated in recent years [5-12], offering diverse tools for the characterization of quantum optics processes and for the verification or calibration of theoretical models for atom-photon interactions. In many cases, the measurements themselves or the rotations of the measured axes perturb or even erase the measured state. In other cases, the requirements of the probing protocol contrast with the operating conditions of the measured system, e.g. an application of a magnetic field in a magnetically-sensitive arrangement [13]. A continuous monitoring of the state dynamics requires an interrogation method that does not substantially damage it, which is possible for non-radiating systems using weak all-optical probing. Recently, all-optical measurements of dark-resonance spectra have been used to extract the ground-state populations of cold rubidium atoms [10]. However, since dark resonances are twophoton transitions requiring non-linear pumping of a certain dark state, they cannot be carried out with very weak probe beams. Here we propose an all-optical linear interrogation method with essentially no lower bound for the probe intensity. Having a negligible effect on the ensemble, the procedure can be carried out side by side with other processes.

In 14], re-population processes within the groundstate manifold of a thermal rubidium vapor were investigated using a series of weak light pulses of single frequency and polarization. The time-dependent absorption was analyzed to estimate the ground-state decay and decoherence rates. The scheme presented here generalizes this method for multiple frequencies and polarizations, in order to characterize the detailed sub-level population distribution within the ground manifold.

The populations of the different angular-mumentum states within the ground level of an atomic ensemble can be readily measured in a spectroscopic measurement, only when the Zeeman and the hyperfine splittings are both larger than the spectral widths of the transitions. In a room-temperature atomic vapor, the optical Doppler broadening is on the order of hundreds of $\mathrm{MHz}$, and the collisional (pressure) broadening in a typical case of a buffer-gas environment may even exceed that. Thus, the magnetic lines are resolved only for magnetic fields much larger than 100 Gauss, which are not suitable in many setups, such as storage-of-light [15], atomic frequency references [2, 16], magnetometry [17], and optical pumping [18. In these situations the angular-mumetum states are degenerate with respect to the measurement. We show that this effective degeneracy may be lifted utilizing the differences in the transition strengths for each state and each polarization. Therefore in contrast to previous schemes, the reconstruction can be carried out even when the contribution of any single state in each measurement could not be distinguished, and thus no application of a significant external magnetic field is required. The basic concepts of the reconstruction method are outlined in Sec. II, and the experimental setup of the probing system is described in Sec. III. The results for various optical-pumping experiments are presented in Sec. IV. 


\section{THEORY OF POPULATION RECONSTRUCTION}

We study optical atomic transitions of the form $\left|F, m_{F}\right\rangle \rightarrow\left|F^{\prime}, m_{F^{\prime}}\right\rangle$, where $F$ and $m_{F}$ are respectively the hyperfine and Zeeman numbers within the groundlevel manifold, $F^{\prime}$ and $m_{F^{\prime}}$ denote the excited manifold. For the sake of simplicity, we consider here only circularly-polarized light, with $m_{F^{\prime}}=m_{F} \pm 1$ corresponding to the $\sigma^{ \pm}$polarizations. The absorption of a weak probe field due to each atomic transition depends on the inherent strength of the transition, determined by the electric dipole moment, and on the frequency detuning from the resonance. The absorption coefficient is thus written as the sum over the contributions of all possible transitions,

$$
\alpha^{ \pm}(\omega)=\sum_{\left|F, m_{F}\right\rangle} C_{\left|F, m_{F}\right\rangle}^{ \pm}(\omega) \times P_{\left|F, m_{F}\right\rangle},
$$

where

$$
C_{\left|F, m_{F}\right\rangle}^{ \pm}(\omega)=n_{0} \frac{4 \pi \omega}{c \hbar} \sum_{F^{\prime}} K_{F, m_{F}}^{F^{\prime}, m_{F} \pm 1}(\omega)\left(\mu_{F, m_{F}}^{F^{\prime}, m_{F} \pm 1}\right)^{2}
$$

is the excitation spectrum of a specific ground-level state $\left(\left|F, m_{F}\right\rangle\right), P_{\left|F, m_{F}\right\rangle}$ is its population, and $n_{0}$ is the atomic density. The dipole matrix element is expressed in terms of the Clebsch-Gordan coefficients as 19 ]

$$
\begin{gathered}
\mu_{F, m_{F}}^{F^{\prime}, m_{F} \pm 1}=\mu_{0} \sqrt{2 F^{\prime}+1}\left\{\begin{array}{ccc}
J & J^{\prime} & 1 \\
F & F^{\prime} & I
\end{array}\right\} \times \\
\left\langle F, m_{F}, 1, \pm 1 \mid F^{\prime}, m_{F} \pm 1\right\rangle,
\end{gathered}
$$

where $\mu_{0}$ factors out the coefficients common to all transitions, $J$ and $J^{\prime}$ are the total electronic angular momenta of the ground and excited levels, respectively, and $I$ is the nuclear angular momentum. The frequency dependence of the spectrum is introduced via the Voigt functions, $K_{F, m_{F}}^{F^{\prime}, m_{F} \pm 1}(\omega)$, centered around the resonance frequencies of $\left|F, m_{F}\right\rangle \rightarrow\left|F^{\prime}, m_{F^{\prime}}\right\rangle$ and accounting for both the homogeneous and the inhomogeneous broadening components. Sampling the spectra of the $\sigma^{+}$and $\sigma^{-}$ polarizations at $N$ different frequencies transforms Eq. (1) into a matrix form,

$$
\alpha_{(2 N \times 1)}=\overline{\mathbf{C}}_{(2 N \times M)} \cdot \mathbf{P}_{(M \times 1)},
$$

where each row corresponds to a single measurement, $\alpha$ is the vector of measured absorption coefficients, $\mathbf{P}$ is the ground-state populations, and $\overline{\mathbf{C}}$ is the coupling matrix obtained from Eq. (2). The population reconstruction is thus possible via a matrix inversion, providing that at least $M$ linearily-indpendent measurements are made. A least-squares fit to the data with $2 N \gg M$ measurements would increase the accuracy and robustness of the reconstruction, and is more adequate in the presence of real-life measurement noise and systematic errors. Such a fit to Eq.(4) is given by

$$
\mathbf{P}=\overline{\mathbf{C}}^{+} \alpha,
$$

where $\overline{\mathbf{C}}^{+}=\left(\overline{\mathbf{C}}^{\mathbf{T}} \overline{\mathbf{C}}\right)^{-1} \overline{\mathbf{C}}^{\mathbf{T}}$ is the Moore-Penrose pseudoinverse [20]. Finally, if the atomic density, $n_{0}$, is not known a priori within the desired accuracy, it should be determined by the constrain $\sum P_{\left|F, m_{F}\right\rangle}=1$. In practice, this can be achieved directly from the measured spectra using the relation

$$
\int d \omega\left[\alpha^{+}(\omega)+\alpha^{-}(\omega)\right]=Q n_{0} \frac{4 \pi \omega}{c \hbar},
$$

where the identity $\sum_{F^{\prime}}\left[\left(\mu^{+}\right)^{2}+\left(\mu^{-}\right)^{2}\right]=Q(Q=2 / 3$ for rubidium-87 D1 transitions) and $\int d \omega K(\omega)=1$ are used.

In order to demonstrate the strengths and the limitations of our scheme, we hereafter concentrate on the D1 transition of rubidium- 87 . The ground and upper manifolds of this transition consist of two hyperfine levels, $F=1,2$, which in turn are Zeeman splitted, as depicted in Fig 1p. When the Zeeman and the hyperfine splittings are larger than the spectral line-width, it is easy to show that $\overline{\mathbf{C}}^{\mathbf{T}} \overline{\mathbf{C}}$ is nearly diagonal and trivially invertible, and the populations of the eight ground-level states are immediately obtained from Eq.(5). In the opposite limit where both splittings are small, and the spectral lines are indistinguishable, two of the eigenvalues of $\overline{\mathbf{C}}^{\mathbf{T}} \overline{\mathbf{C}}$ in Eq.(5) are close to zero due to the Clebsch-Gordan properties [27]. For this limiting case, our reconstruction scheme becomes sensitive to noise and errors and thus not applicable.

Nevertheless in the most common intermediate regime, the Zeeman sublevels are degenerate but the ground and excited hyperfine sublevles are spectrally resolved. For a thermal rubidium vapor, the ground-level hyperfine splitting is much larger than the spectral line-width $(6.8 \mathrm{GHz}$ compared with $\sim 500 \mathrm{MHz}$ ), and the absorption spectra of the $F=1$ and the $F=2$ manifolds are completely separable. The spectrum of each $F$ manifold, for a given probe polarization $q$, provides two meaningful data points, $\xi_{F}^{F^{\prime}=1, q}$ and $\xi_{F}^{F^{\prime}=2, q}$, which are the sum of all (Zeeman) transitions between $F$ and $F^{\prime}$. A fitting process $\alpha^{q}(\omega)=\sum_{F, F^{\prime}=1,2} \xi_{F}^{F^{\prime}, q} K_{F}^{F^{\prime}}(\omega)$, where $K_{F}^{F^{\prime}}(\omega)$ are the degenerate Voigt profiles, gives $\xi_{F}^{F^{\prime}, q}$ with high accuracy. With $\xi_{F}^{F^{\prime}, q}$ known, one is left with the following reduced equation,

$$
\left.\xi_{F}^{F^{\prime}, q}=\sum_{\left|F, m_{F}\right\rangle}\left(\mu_{F, m_{F}}^{F^{\prime}, m_{F} \pm q}\right)^{2} P_{\left|F, m_{F}\right\rangle}\right\rangle
$$

for each ground-state manifold. For example, using circular polarizations $\sigma^{ \pm}$, a rank-3 matrix,

$$
\left(\begin{array}{l}
\xi_{1}^{1,+} \\
\xi_{1}^{2,+} \\
\xi_{1}^{1,-} \\
\xi_{1}^{2,-}
\end{array}\right)=\left(\begin{array}{ccc}
1 / 12 & 1 / 12 & 0 \\
1 / 12 & 1 / 4 & 1 / 2 \\
0 & 1 / 12 & 1 / 12 \\
1 / 2 & 1 / 4 & 1 / 12
\end{array}\right)\left(\begin{array}{c}
P_{|1,-1\rangle} \\
P_{|1,0\rangle} \\
P_{|1,+1\rangle}
\end{array}\right),
$$

is obtained for the $F=1$ manifold, with a solution

$$
\left(\begin{array}{c}
P_{|1,-1\rangle} \\
P_{|1,0\rangle} \\
P_{|1,+1\rangle}
\end{array}\right)=3\left(\begin{array}{ccc}
3 & 1 & -6 \\
1 & -1 & 6 \\
-1 & 1 & -2
\end{array}\right)\left(\begin{array}{l}
\xi_{1}^{1,+} \\
\xi_{1}^{2,+} \\
\xi_{1}^{1,-}
\end{array}\right) .
$$




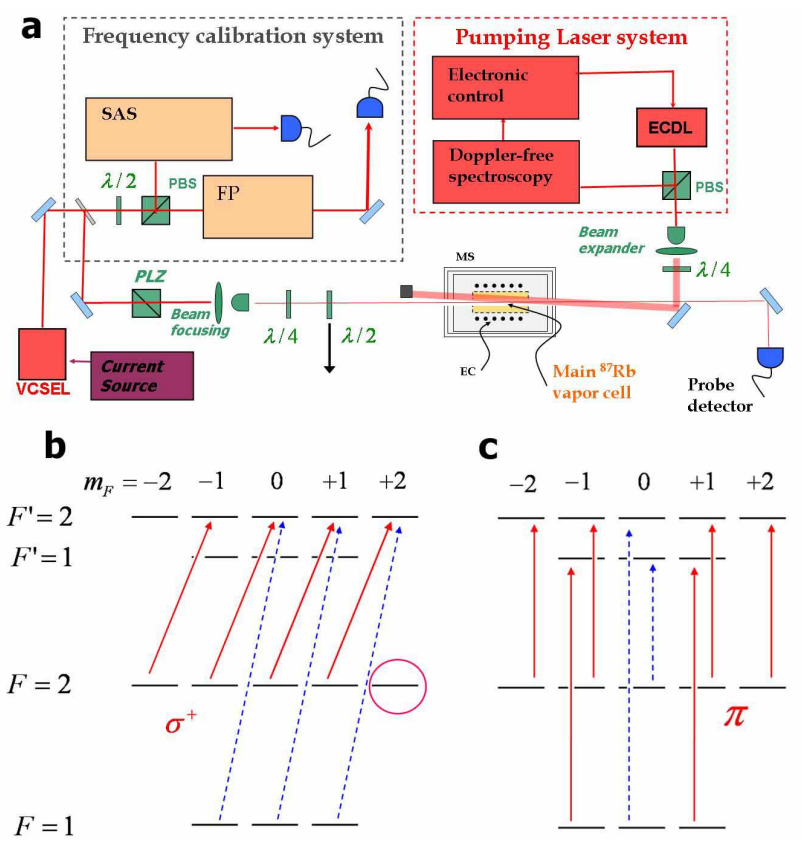

FIG. 1: (color online). The setup of experiment I: a. VCSEL - vertical-cavity surface-emitting laser diode; PBSpolarizing beam splitter; PLZ - GLAN polarizer; MSmagnetic shield, EC-electric coil; ECDL-external-cavity diode laser. b. Energy levels in experiment I, and exciting pump fields, showing resonant transitions in red solid lines and non-resonant transition in blue dashed lines. The endstate $\left|F=2, m_{F}=+2\right\rangle$ is circled. c. Energy levels in experiment II and exciting pump fields.

For the $F=2$ manifold, the matrix obtained is also of rank 3 , whereas the manifold contains 5 sublevels, thus preventing the inversion. The addition of other excitations, either to different levels (e.g., the D2 transitions) or with a $\pi$-plorization, are ineffective in resolving this case. This follows from the symmetry properties of the dipole matrix elements, namely the absorption of an isotropic light field [19] and the spherical behavior of the dipole moment,

$$
\mu_{F, m_{F}}^{F^{\prime}, m_{F}-q}=\mu_{F,\left(-m_{F}\right)}^{F^{\prime},\left(-m_{F}\right)+q}
$$

enabling no more than three independent relations. In conclusion, the detailed populations of the $F=1$ manifold, i.e. $P_{|1,-1\rangle}, P_{|1,0\rangle}$, and $P_{|1,+1\rangle}$, can be recovered, but the $F=2$ populations can not. The total population in the $F=2$ manifold is determined, and in specific cases, as we show in what follows, the total $F=2$ population can be related to that of the maximally-polarized states $|2, \pm 2\rangle$, in effect reconstructing the complete ground-level population distribution.

\section{EXPERIMENTAL SETUP}

The experimental arrangement, with both the pumping and the probing setups, is depicted in Fig. 11. We drive the atomic ensemble to a specific atomic state using a pump beam, as detailed in sections IV A and IV B. Simultaneously with the pumping process, we employ a continuous weak light field for probing the medium (Fig. 13). For the probe beam, we use a vertical-cavity surface-emitting laser diode (VCSEL), and its frequency is scanned by ramping the current. The probe beam, typically of $\sim 1 \mathrm{~mm}$ diameter and $\sim 50 \mathrm{nW}$ power, traverses a Glan-Laser polarizer followed by a quarter-wave plate, to produce a $\sigma^{+}$polarization. A half-wave plate, mounted on a flip-flop, is used to convert the probe polarization to $\sigma^{-}$in alternating measurements. The probe then passes through the main vapor cell and is measured by a photodiode. The frequency scans are of $\sim 12 \mathrm{GHz}$ and carried out at a repetition rate which is sufficiently small in order to probe the stady-state distribution $(12.5 \mathrm{~Hz})$. Each scan consists of 2500 data points (See $N$ in Eq. (41)). The population reconstruction scheme studied here requires high accuracy determination of the frequency of the probe. To avoid errors caused by frequency drifts of the VCSEL, and nonlineartities in the frequency scan, a frequency calibration system was utilized. An accurate frequency scale was acquired by a saturated absorption spectroscopy setup [21] in a reference vapor cell. Nonlinearities in the frequency scan were corrected using frequency markers produced by a Fabry-Perot cavity [22]. After the frequency calibration process, the absorption spectra were introduced into Eq. (5) and regressed with a numerical code.

\section{EXPERIMENTAL RESULTS}

\section{A. Experiment I: Optical pumping with $\sigma^{+}$ polarized light}

In the first experiment, we pump the atomic ensemble with an external-cavity diode laser (ECDL), stabilized to the $F=2 \rightarrow F^{\prime}=2$ transition of the D1 manifold (Fig. 10). As illustrated in Fig. 17a, the pumping laser passes through a polarizing beam splitter (PBS) followed by a quarter-wave plate to produce the circular polarization. The beam is then directed into the 5 -cm long main vapor cell, containing isotopically pure ${ }^{87} \mathrm{Rb}$ and 10 Torrs of neon buffer gas. The cell is at room temperature, providing a vapor density of $\sim 10^{10} / \mathrm{cc}$. The cell is located within a three-layered magnetic shield, and an electric coil produces a small axial magnetic field $\left(B_{z}=450 \mathrm{mG}\right)$ to set the quantization axis.

We have performed population-distribution measurements with this arrangement, as described in Sec. III, for various pump powers. Figure 2 depicts probe absorption curves for three typical pump powers. When no pump is applied (panel b in Fig. 2) the medium is expected 


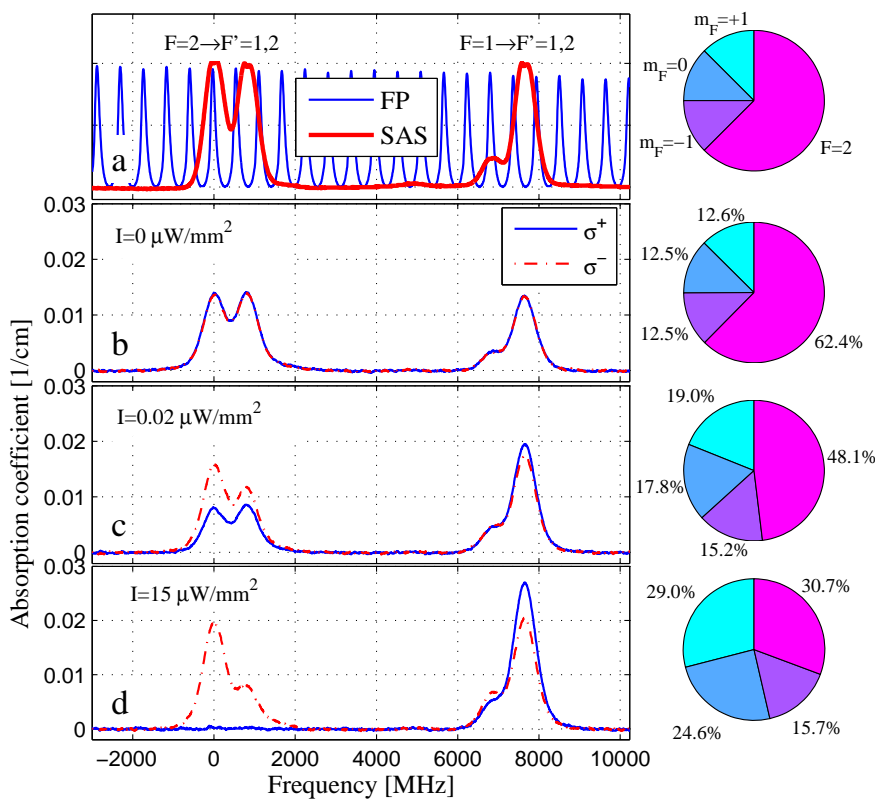

FIG. 2: (color online) Measured signals in experiment I: a. FP signal (blue thin lines) and SAS signal (red thick line) are used for callibrating the frequency axis. (b,c,d) Absorption spectra measured with $\sigma^{+}$polarization (solid lines) and $\sigma^{-}$ polarization (dashed lines) with various pump powers, showing the thermal equilbrium (b), depopulated $F=2$ (c), and vacancy of the $m_{F} \leq 1$ states in $F=2(\mathrm{~d})$. On the right are the population distribution of the $F=1$ manifold and the total population of $F=2$ manifold.

to be in thermal equilibrium, in which the population is equally distributed among the eight levels of the groundstate. Indeed, the measured $F=1$ populations are equal up to the measurment error. As the pump power is increased, the absorption from the $F=2$ manifold is reduced due to the depopulation of the levels within the $F=2$ manifold. The $\sigma^{+}$and $\sigma^{-}$absorption curves differ as a consequence of the increasing polarization of the medium. For pump intensities larger than $10 \mu \mathrm{W} / \mathrm{mm}^{2}$, the $\sigma^{+}$absorption from the $F=2$ manifold nullifies, showing that the $\left|F=2, m_{F}=-2,-1,0,+1\right\rangle$ levels are vacant.

The populations of the three states within the groundstate $F=1$ manifold are reconstructed using the procedure described in Sec. [II The best-fitted values of the homogeneous and inhomogeneous broadenings of the Voigt lineshape, $\Gamma=103 \mathrm{MHz}$ and $\sigma=202 \mathrm{MHz}$ (FWHM $\sim 600 \mathrm{MHz}$ ), are as expected for our room-temperature buffer-gas system. Although the population distribution of the $F=2$ manifold is not formally solved, a leastsquares minimization process assuming non-negative values can be used to estimate the detailed populations within the $F=2$ manifold. At high pump powers, in which the population of $F=2$ is associated with $\left|F=2, m_{F}=+2\right\rangle$ only, this estimation becomes reliable

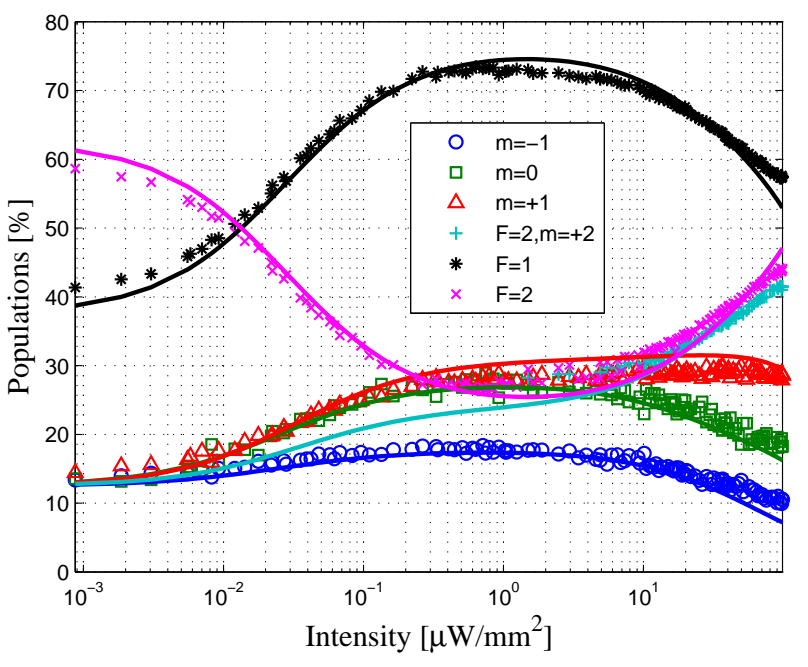

FIG. 3: The reconstructed populations versus five decads of the pump intensity in experiment I (symbols). The numerical model (lines) agrees well with the measurements.

and the populations of all eight states within the groundlevel are extracted. For the strongest pump used in our experiment, over $95 \%$ of the $F=2$ population piles up in the $m_{F}=+2$ level. The reconstructed population distribution is shown in the inset of each panel in Fig. 2,

The detailed population measurements provide a substantial dataset, which can be used to benchmark a theoretical multilevel model [28]. In Fig. 3 we show a comparison between the experimental measurements and the results of our multilevel numerical model, for a wide range of pump powers. The fitted Voigt parameters of the measured spectra $(\Gamma$ and $\sigma$ ) were used as an input to the model. A single model parameter that determines the ground-level population equilibration was fitted to the data, effectivly describing the diffusion-induced decay and wall collisions (decay due to spin-exchange collisions is negligible in our conditions). It is evident that our model describes well the population distribution within the ground levels for five decades of pump power.

When the pump power is very weak a thermal distribution within the ground-state is observed. As the pump power is increased, the medium is polarized and more population accumulates in the higher $m_{F}$ levels of the $F=1$ manifold (triangles and squares in Fig. 3). At higher pump powers, the total population of the $F=1$ manifold is depleted due to off-resonant pumping. All these phenomena are well described by the multilevel model with a single fit parameter. Remaining discrepancies result from magnetic field inhomogeneity and from residual ${ }^{85} \mathrm{Rb}$ isotope in the cell (about $2 \%$ ). It is interesting to note that an extremely efficient pumping to the maximally polarized state, $\left|F=2, m_{F}=2\right\rangle$, can be achieved by tuning the frequency of the pumping laser in between the ground-level hyperfine transitions. We 
verified this experimentally by locking the laser to the $F=3 \rightarrow F^{\prime}=2$ transition of ${ }^{85} \mathrm{Rb}$, and found that more than $90 \%$ of the ground-level population was pumped to the maximally polarized state for a pump intensity of about $100 \mu \mathrm{W} / \mathrm{mm}^{2}$.

\section{B. Experiment II: Optical pumping with $\pi$ polarized light}

We now turn to implement our reconstruction method for the case of CPT-based atomic frequency reference. One of the well known limitations of these frequency references is optical pumping of the atomic population from the clock-states $\left|F=1, m_{F}=0\right\rangle$ and $\left|F=2, m_{F}=0\right\rangle$ [3]. An approach previously investigated to resolve this problem is the repopulation of the clock states using additional $\pi$-polarized light field [23, 24]. To study this repopulation technique, we pump the ensemble with a RF-modulated $\pi$-polarized field, as depicted in Fig. 1;. We use a current-modulated VCSEL at a modulation frequency of 3.0 GHz. The laser frequency is tuned such that the +1 and -1 sidebands are resonant with the $F=1 \rightarrow F^{\prime}=1$ and $F=2 \rightarrow F^{\prime}=2$ transitions, respectively. The laser is linearly polarized with a PBS and directed into a vapor cell containing isotopically pure ${ }^{87} \mathrm{Rb}$ and 10 Torr of nitrogen buffer gas, at temperature of $58^{\circ} \mathrm{C}$. The vapor cell is magnetically shielded and a constant magnetic field of about $100 \mathrm{mG}$ is applied to set the quantization axis along the pump beam polarization direction (to induce $\pi$ transitions). The probe beam, used for the population distribution measurement, is applied along the magnetic field axis.

Fig. 4 presents the reconstructed populations as well as the calculated results of our multilevel model. The $\left|F=1, m_{F}=0\right\rangle$ clock-state accumulates excess population as the pump power is increased, reaching a maximal population of twice the thermal equilibrium population. The populations in the $m_{F}= \pm 1$ states show symmetric behavior, demonstrating that a pure $\pi$-pumping was applied to the sample. As the pump power is increased, the population of the $F=2$ manifold decreases, since the $F=2 \rightarrow F^{\prime}$ transitions are more efficient than the $F=1 \rightarrow F^{\prime}$ transitions. To emphasize the destructive effect of the non-resonant excitations (blue dashed arrows in Fig. 1:), we have performed an additional calculation, in which the non-resonant excitations were excluded (dashed lines in Fig. 44). It is evident that the non-resonant excitations pose a limit on the efficiency of the $\pi$ re-pumping technique.

\section{CONCLUSIONS}

We have presented an all-optical scheme to measure the populations within the ground-state of multilevel atoms and have demonstrated its implementation for a ${ }^{87} \mathrm{Rb}$ vapor. In our scheme, the required light and mag-

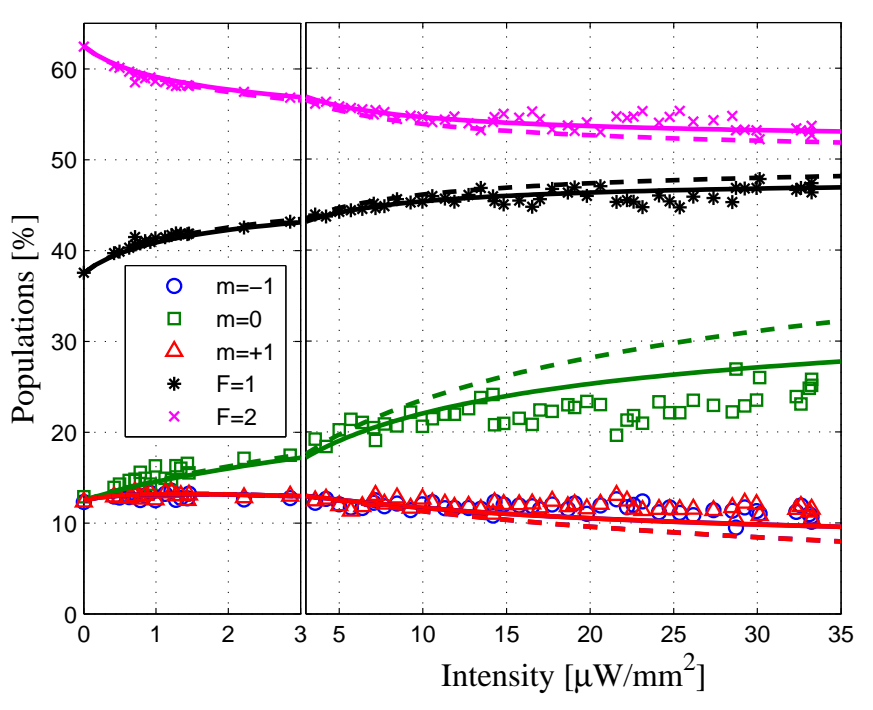

FIG. 4: (color online) The reconstructed populations versus the pump intensity in experiment II, showing the agreement between the measured population (symbols), a numerical model (solid lines) and a numerical model that neglects non-resonant transitions (dashed lines)

netic fields are weak, and the degeneracy created by the optical-transitions width is lifted by utilizing the properties of the dipole matrix elements. As an intuitive quantity characterizing the state of the ensemble, the population distribution is most natural in monitoring dynamic processes, and in analyzing the initial quantum state in precise experiments [25]. For the D1 transition of ${ }^{87} \mathrm{Rb}$, the scheme reconstructs the detailed population distribution of the $F=1$ manifold and the total population of the $F=2$ manifold. In the framework of linear spectroscopic measurements, the population distribution of the $F=2$ level is fully reconstructed only if one can relate the total population of the $F=2$ manifold to one of the maximally polarized states. In order to reconstruct the full density matrix, a tomographic scheme (as in [5]) could be applied. For that, the population distribution measurements, as presented in this work, should be carried out along $4 F+1$ different directions. We have used the scheme in two optical-pumping experiments and demonstrated its strength in describing various optical processes, such as resonant and non-resonant excitations and depolarization, and in verifying and calibrating the parameters of an elaborated numerical model. We further employed the calibrated model to calculate the improvement of the contrast of a CPT based frequency reference.

\section{Acknowledgments}

We wish to acknowledge the helpful discussions with Amnon Fisher, Nir Davidson, Ran Fischer and Asif 
Sinay. We thank Yoav Erlich for technical support.

[1] E. Arimondo, "Coherent Population Trapping in Laser Spectroscopy", Progress in Optics (Elsevier, Amsterdam, 1996), vol. 35.

[2] S. Knappe, V. Shah, P. D. D. Schwindt, L. Hollberg, J. Kitching, L.-A. Liew, and J. Moreland, Applied Physics Letters 85, 1460 (2004), URL http://link.aip.org/link/?APL/85/1460/1

[3] J. Vanier, M. W. Levine, D. Janssen, and M. Delaney, Phys. Rev. A 67, 065801 (pages 4) (2003).

[4] Y.-Y. Jau, A. B. Post, N. N. Kuzma, A. M. Braun, M. V. Romalis, and W. Happer, Phys. Rev. Lett. 92, 110801 (2004).

[5] G. Klose, G. Smith, and P. S. Jessen, Phys. Rev. Lett. 86, 4721 (2001).

[6] D. T. Smithey, M. Beck, M. G. Raymer, and A. Faridani, Phys. Rev. Lett. 70, 1244 (1993).

[7] T. J. Dunn, I. A. Walmsley, and S. Mukamel, Phys. Rev. Lett. 74, 884 (1995).

[8] D. Leibfried, D. M. Meekhof, B. E. King, C. Monroe, W. M. Itano, and D. J. Wineland, Phys. Rev. Lett. 77, 4281 (1996).

[9] I. L. Chuang, N. Gershenfeld, and M. Kubinec, Phys. Rev. Lett. 80, 3408 (1998).

[10] B. Wang, Y. Han, J. Xiao, X. Yang, C. Zhang, H. Wang, M. Xiao, and K. Peng, Phys. Rev. A 75, 051801 (2007).

[11] A. Silberfarb, P. S. Jessen, and I. H. Deutsch, Phys. Rev. Lett. 95, 030402 (2005).

[12] G. A. Smith, A. Silberfarb, I. H. Deutsch, and P. S. Jessen, Physical Review Letters 97, 180403 (pages 4) (2006), URL http://link.aps.org/abstract/PRL/v97/e180403

[13] J. C. Allred, R. N. Lyman, T. W. Kornack, and M. V. Romalis, Phys. Rev. Lett. 89, 130801 (2002).

[14] M. Shuker, O. Firstenberg, Y. Sagi, A. Ben-kish, N. Davidson, and A. Ron, Phys. Rev. A 78, 063818 (2008).

[15] M. D. Lukin, Rev. Mod. Phys. 75, 457 (2003).

[16] N. Cyr, M. Tetu, and M. Breton, IEEE Transactions on Instrumentation and Measurement 42, 640 (1993).

[17] P. D. D. Schwindt, S. Knappe, V. Shah, L. Hollberg, and
J. Kitching, Applied Physics Letters 85, 6409 (2004).

[18] W. Happer, Rev. Mod. Phys. 44, 169 (1972).

[19] D. A. Steck, 'Rubidium 87 D Line Data', available online at http://steck.us/alkalidata (revision 2.1.2, 12 August 2009).

[20] W. H. Press, S. A. Teukolsky, W. T. Vetterling, and B. P. Flannery, in Numerical Recipes (Cambridge University Press, Cambridge, 1992).

[21] D. W. Preston, American Journal of Physics 64, $1432 \quad$ (1996), URL http://link.aip.org/link/?AJP/64/1432/1.

[22] D. Budker, D. J. Orlando, and V. Yashchukc, AJP (1998).

[23] G. Kazakov, I. Mazets, Y. Rozhdestvensky, G. Mileti, J. Delporte, and B. Matisov, Eur. Phys. J. D 35, 445 (2005), URL http://dx.doi.org/10.1140/epjd/e2005-00213-7.

[24] A. Sinay, Master's thesis, Technion-Israel Institute of Technology (2009), URL http://www.graduate.technion.ac.il/Theses/Astracts. asp?Id=2

[25] D. N. Matsukevich, T. Chaneliére, S. D. Jenkins, S.-Y. Lan, T. A. B. Kennedy, and A. Kuzmich, Phys. Rev. Lett. 96, 030405 (2006).

[26] R. Fischer, O. Firstenberg, M. Shuker, and A. Ron, Opt. Express 17, 16776 (2009), URL http://www . opticsexpress.org/abstract. cfm?URI=oe-17-19-1677

[27] The two eigenvalues remain small also if absorption measurements of a $\pi$-polarized probe are added to the reconstruction procedure.

[28] Our numerical model describes the dynamics and the steady-state solutions of the atomic density-matrix, within a Master-equation formalism, and was previously used in [14, 26]. The model incorporates all relevant sublevels, e.g. the 16 sublevels of the rubidium-87 D1 transition, and resonant coupling fields. Decay and decoherence processes, within the ground and the excited manifolds and between them, are effectively described by a suitable Lindblad form. We use 11 velocity groups to model the doppler broadening. 